Portland State University

PDXScholar

Mechanical and Materials Engineering Faculty

Publications and Presentations

4-2018

\title{
The Role of Surface Vorticity during Unsteady Separation
}

\author{
Matthew Scott Melius \\ Portland State University, meliusms@gmail.com \\ Karen Mulleners \\ École Polytechnique Fédérale de Lausanne \\ Raul Bayoan Cal \\ Portland State University, rcal@pdx.edu
}

Follow this and additional works at: https://pdxscholar.library.pdx.edu/mengin_fac

Part of the Fluid Dynamics Commons

Let us know how access to this document benefits you.

\section{Citation Details}

Melius, Matthew Scott; Mulleners, Karen; and Cal, Raul Bayoan, "The Role of Surface Vorticity during Unsteady Separation" (2018). Mechanical and Materials Engineering Faculty Publications and Presentations. 202.

https://pdxscholar.library.pdx.edu/mengin_fac/202

This Article is brought to you for free and open access. It has been accepted for inclusion in Mechanical and Materials Engineering Faculty Publications and Presentations by an authorized administrator of PDXScholar. Please contact us if we can make this document more accessible: pdxscholar@pdx.edu. 


\title{
The role of surface vorticity during unsteady separation
}

\author{
Matthew S. Melius, ${ }^{1, a)}$ Karen Mulleners, ${ }^{2}$ and Raúl Bayoán Cal ${ }^{1}$ \\ ${ }^{1}$ Portland State University, Portland, Oregon 97201, USA \\ ${ }^{2}$ École Polytechnique Fédérale de Lausanne, 1015 Lausanne, Switzerland
}

(Received 26 September 2017; accepted 22 March 2018; published online 25 April 2018)

\begin{abstract}
Unsteady flow separation in rotationally augmented flow fields plays a significant role in a variety of fundamental flows. Through the use of time-resolved particle image velocimetry, vorticity accumulation and vortex shedding during unsteady separation over a three-dimensional airfoil are examined. The results of the study describe the critical role of surface vorticity accumulation during unsteady separation and reattachment. Through evaluation of the unsteady characteristics of the shear layer, it is demonstrated that the buildup and shedding of surface vorticity directly influence the dynamic changes of the separation point location. The quantitative characterization of surface vorticity and shear layer stability enables improved aerodynamic designs and has a broad impact within the field of unsteady fluid dynamics. Published by AIP Publishing. https://doi.org/10.1063/1.5006527
\end{abstract}

\section{INTRODUCTION}

Unsteady flow separation is highly complex and represents an important topic of investigation in the field of fluid mechanics. The extent of separation and time scales over which it occurs is a fundamental problem which is not yet fully understood. Unsteady separation is a common phenomenon in natural and industrial applications such as divergent flows in the bifurcation of a carotid artery when blood flow to the brain is constricted, ${ }^{1}$ biological and bio-inspired flight, ${ }^{2}$ helicopters in forward flight, jet engines, hydroelectric turbines, and wind turbines. Unsteady separation in aerodynamics is accompanied with the erratic movement of the separation point location which causes highly dynamic and unpredictable loads on airfoils. ${ }^{3}$

Separation conditions for steady flow past a twodimensional streamlined body, as proposed by Prandtl, state that flow will separate from the surface where the skin-friction is reduced to zero and a negative pressure gradient exists. This gives an Eulerian description of the boundary layer behavior and fits well in the case of steady separation. ${ }^{4}$ In the case of unsteady flow separation, a singularity appears in the steady boundary layer solution when the wall shear stress vanishes. ${ }^{5}$ This so-called Goldstein singularity was studied simultaneously by Moore, ${ }^{6}$ Rott, ${ }^{7}$ and Sears, ${ }^{8}$ all independently arriving at the conclusion that vanishing wall-shear stress and the accompanying flow reversal near a wall do not necessarily guarantee flow separation under unsteady conditions, which became known as the Moore, Rott, and Sears (MRS) criterion. According to the MRS criterion, the separation point must occur on the zero vorticity line bisecting a recirculation region.

To address the ambiguity associated with unsteady separation resulting from the MRS criterion, a Lagrangian solution was proposed by van Dommelen and Shen. ${ }^{9}$ The Lagrangian approach was found to accurately describe the convergence

a)meliusms@gmail.com and ejection of fluid particles from an unsteady boundary layer flow. The Lagrangian formulation for the unsteady boundary layer has two advantages: (i) It decouples the motion in the streamwise direction from that in the wall-normal direction and (ii) it offers an objective criterion for boundary layer separation. Recently, Surana et al. ${ }^{10}$ developed an exact theory of steady flow separation and reattachment on boundaries at rest within a Lagrangian framework, which has been extended to include unsteady separation. Surana et al. ${ }^{10}$ focused on the connection between the Lagrangian sources of flow separation and the Eulerian fields of skin-friction lines, i.e., the separation lines, by analyzing unstable manifolds that originate at the surface. The unstable manifolds are lines or surfaces that attract and eject fluid particles away from the boundary. ${ }^{10}$ The method for manifold identification presented by Farazmand and Haller ${ }^{11}$ allows for the calculation of Lagrangian coherent structures (LCS) from Eulerian velocity fields. To improve future predictive capabilities of the onset and duration of unsteady separation, more insight into the role of vorticity in the shear layer that develops at the interface between the outer flow and a separated flow region near the suction surface of the airfoil is desirable. This shear layer experiences spatial perturbations following the Kelvin-Helmholtz instability. These perturbations increase with increasing extent of the separated flow region and grow in magnitude as they are convected downstream. ${ }^{12}$ As a result of the instability, the shear layer undulates and the flow near its apex accelerates while the pressure reduces. Near the troughs of the shear layer, the flow decelerates and the pressure increases, thus leading to an amplification of the shear layer wave downstream. ${ }^{13}$ The growth of the shear layer perturbations is also connected to inflections of the shear layer directly downstream of the separation point and abrupt movements of the separation point location itself. ${ }^{14}$ The shear layer perturbation amplitude at the point of separation is expected to have a direct effect on the eruption of near-wall vorticity away from the surface into the outer flow. ${ }^{3}$ Despite the advances in the theoretical description of unsteady separation, there is still need for 
experimental analysis of the role of vorticity at the surface of an airfoil in the context of unsteady separation. Identification of the initial separation location and strength of separating shear layers is an important challenge in various engineering applications. ${ }^{15}$

This study provides experimental evidence of the critical role of surface vorticity and its accumulation during unsteady separation and reattachment on a scaled three-dimensional wind turbine blade. Time-resolved velocity field measurements by means of particle image velocimetry (PIV) were conducted in a cross-sectional plane near the root of the model blade which was subjected to an unsteady variation in the angle of attack mimicking a gust encounter. The angle of attack variation included a pitch up motion from an angle of attack below the static stall limit to an angle of attack well above that limit at which the flow is fully separated. After a short pause, the angle of attack was reduced again to its initial value at which the flow is attached again. The pitch down rate had the same magnitude but opposite sign as the pitch up rate. The flow development during the transition of the flow from attached to separated and back to attached was recorded and analyzed. In particular, the separation point location, near wall vorticity, and shear layer characteristics are tracked in time. The causal relationship between the accumulation and shedding of surface vorticity and the dynamic changes in the separation point location is further explored.

\section{EXPERIMENTAL SETUP}

Experiments were conducted in the closed $2 \mathrm{~m} \times 1.8 \mathrm{~m}$ test section of the closed loop wind tunnel at Deutsche Forschungsanstalt fur Luft und Raumfahrt (DLR) in Göttingen. An aluminum model of the NREL $5 \mathrm{MW}$ wind turbine blade is manufactured at 1:63 scale with a total span of $1.05 \mathrm{~m}$. The chord length is doubled while maintaining the thickness-to-chord ratio and spanwise profile locations to preserve the geometric influence on the suction side pressure gradient. The free stream velocity is set to $U_{\infty}=40 \mathrm{~m} / \mathrm{s}$ with an average turbulence intensity of less than $1 \%$. Velocity field measurements are collected at a radial location of $r / R=0.317$, considered to be within the root section of the blade which was at $h_{p}=0.32 \mathrm{~m}$ above the wind tunnel floor (Fig. 1). At

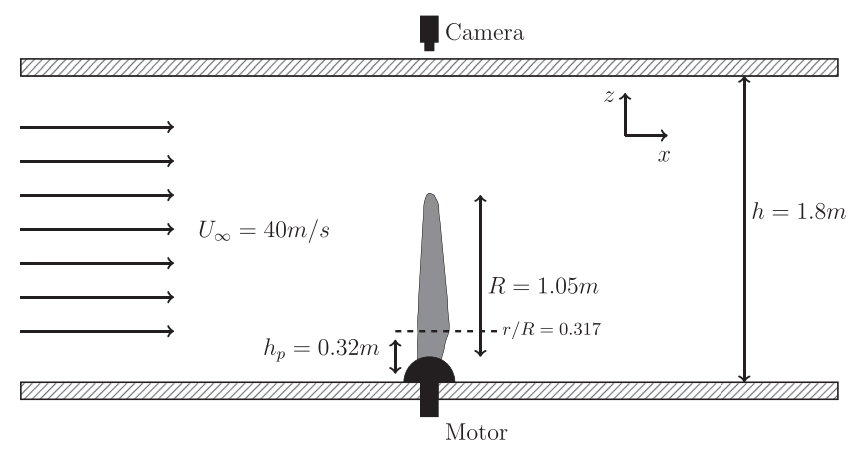

FIG. 1. Experimental setup: scaled wind turbine blade positioned vertically in the $2 \mathrm{~m} \times 1.8 \mathrm{~m}$ test section, with a camera and pitching motor located directly above and below the rotor blade, respectively (figure not to scale). The measurement plane is at $h_{p}=0.32 \mathrm{~m}$, and the free stream velocity is $U_{\infty}=40 \mathrm{~m} / \mathrm{s}$.

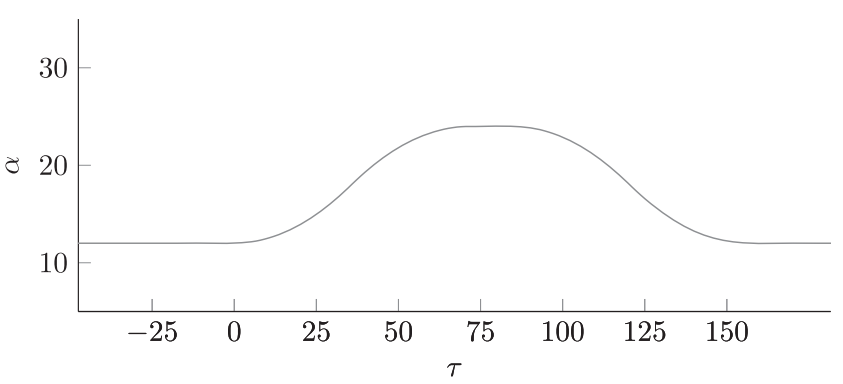

FIG. 2. Experimental pitching cycle, with the angle of attack $\alpha$ on the y-axis and the convective time scale $t U_{\infty} / c, \tau$, on the $\mathrm{x}$-axis.

this location, the cross section is a DU 99-W-350 blade profile. The chord length $c=15 \mathrm{~cm}$. This yields a chord based Reynolds number of $R e_{c}=3.2 \times 10^{5}$. The spanwise twist of the blade is augmented to mimic the effect of rotation on the flow past the turbine blade and is $2.4^{\circ}$ at the measurement location. More details on the selection of the spanwise twist and the experimental blade model are provided in the work of Melius et al. ${ }^{16}$

The blade is pitched dynamically to change its angle of attack from $12^{\circ} \rightarrow 24^{\circ} \rightarrow 12^{\circ}$ (simulating the passage of a single accelerating-decelerating gust) by a stepper motor which is located outside the wind tunnel. A time series of instantaneous velocity snapshots is recorded for a single pitching cycle, as shown in Fig. 2, where $\alpha$ is the angle of attack and $\tau$ is the convective time scale $t U_{\infty} / c$.

To minimize the influence of the wind tunnel wall, the blade base is extended by $10 \mathrm{~cm}$ and is covered by an aerodynamic housing. During the experiment, the blade is pitched with an average pitch rate of $\dot{\alpha}=0.6 \mathrm{~s}^{-1}$. The total blockage created by the blade at the largest angle of attack does not exceed $8 \%$.

Throughout the experiment, time-resolved PIV data are collected at a rate of $1 \mathrm{kHz}$. Images are collected in a crosssectional plane at a radial location of $r / R=0.317$ by a high speed camera located outside of the test section along the pitching axis of the blade. The time-resolved PIV and blade motion are synchronized such that a series of individual measurements cover $0.5 \mathrm{~s}$ before the motion of the blade begins and after completion of the motion cycle to ensure full coverage of the stall cycle. The time series data are collected at a camera resolution of $1744 \mathrm{px} \times 1410 \mathrm{px}$. The measurement window is $254 \mathrm{~mm} \times 205 \mathrm{~mm}$ with a spatial resolution of $6.8 \mathrm{px} / \mathrm{mm}$. The PIV images are processed using an interrogation window size of $32 \mathrm{px} \times 32 \mathrm{px}$ and an overlap of $\approx 80 \%$ yielding a grid spacing of $7 \mathrm{px}$ or $1 \mathrm{~mm}$ which is less than $0.01 \mathrm{c}$. To ensure an acceptable signal-to-noise ratio, the interrogation window size is minimized. The velocity fields are rotated into the airfoil reference system, with the $\mathrm{x}$-axis along the chord and $\mathrm{y}$-axis perpendicular to the chord prior to the analysis.

\section{RESULTS}

To study the interplay between the surface vorticity, the separation point location, and the shear layer instability during unsteady separation and reattachment, the first task is to identify the shear layer and the location of the separation point 
in the individual snapshots. Therefore, attracting LCSs are calculated according to the procedure described by Farazmand and Haller ${ }^{11}$ and the locations of vortical structures are identified by using the $\Gamma$-criterion from the work of Graftieaux et al. ${ }^{17}$ To compute the LCS for a given PIV flow field, the correct velocity and integral time scales must be selected. The integral time scale is calculated based on the length of the shear layer extending from the point of separation and spanning over the remaining chord, defined here as $L=(1-f)$. By dividing $L$ by a characteristic fluctuation velocity, $u^{\prime}$, the result is the integration time, $T=(1-f) * c / u^{\prime}$, which determines the number of time steps used to calculate the LCS. During separation, the characteristic fluctuation velocity is determined to be $u^{\prime}=1.4 \mathrm{~m} / \mathrm{s}$ and the integral spatial scale is $0.05 \mathrm{~m}$ or $x / c=0.33$ which yields an integration time of $0.035 \mathrm{~s}$. These values will change from one stall stage to another.

Figure 3 presents a comparison between the shear layer attracting LCS (black) and a velocity contour at $0.48 U_{\infty}$ (red) for a snapshot of the vorticity field at an angle of attack of $\alpha=24^{\circ}$. The DU 99-W-350 profile is included, and the chordwise $(x)$ and azimuthal $(y)$ axes are normalized by the chord length. Clockwise rotating vortices within the shear layer identified using the $\Gamma$-criterion are shown in orange. The velocity contour at $0.48 U_{\infty}$ practically coincides with the LCS that marks the shear layer as the separating material line between the free stream flow and the separated flow near the airfoil surface. Analysis of the results reveals consistent agreement amongst the shear layer LCS and the velocity contour at $0.48 U_{\infty}$ for the entire stall cycle investigated here. The two shear layer identifiers lie on top of each other until $x / c \approx 0.5$. For $x / c<0.5$, the maximum amplitude of both estimations of the shear layer is nearly equal and both proceed along the shear layer vortex cores. The advantages of using the velocity contour at $0.48 U_{\infty}$ to identify the shear layer are being computationally inexpensive and not requiring time-resolved data to capture accurate shear layer behavior from PIV data. ${ }^{18}$

A Lagrangian separation point was identified as the location at which attracting LCS merge near the airfoil's surface. Due to surface reflections and missing velocity field information close to the surface, the appearance of merging attracting LCS is not consistently observed throughout the experimental results. Comparing the $x / c$ locations of the merging LCS and the first flow reversal, it is found that the difference between

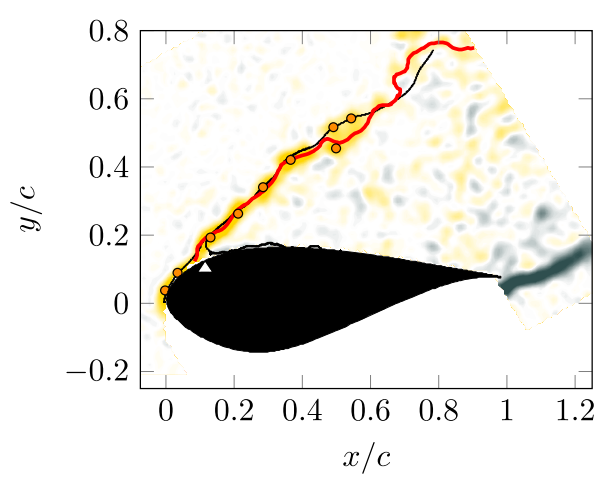

FIG. 3. Velocity ratio $U_{S L} / U_{\infty}=0.48$ (red) plotted with the shear layer attracting LCS (black) on top of the instantaneous vorticity field during full stall at an angle of $\alpha=24^{\circ}$. the two values is less than $0.05 \mathrm{c}$. This Eulerian separation point definition, indicated by the white $\Delta$, is relatively robust, providing data for each time step, and is therefore selected here.

In a next step, the values of vorticity along the shear layer are extracted for all snapshots and local amplitude extrema are determined. The spatial locations of the maximum values of shear layer vorticity for a single snapshot are presented in Fig. 4(a) by white circles, and their normalised magnitudes $\left|\omega^{*}\right|=\left|\omega c / U_{\infty}\right|$ are presented in Fig. 4(b) as a function of the chordwise location $x / c$. The shear layer vorticity $(\omega)$ has a maximum amplitude at or near the separation point $\left|\omega^{*}\right|_{\max }>40$. Further downstream, the vorticity peaks decay exponentially. After applying this technique to all snapshots in the data set, it was observed that the vorticity formed at the separation point is always greater than $\left|\omega^{*}\right|_{\max }=20$. Above this value, the accumulated vorticity entrains enough fluid near the surface to create flow reversal which results in the release of a small-scale vortex. The vorticity magnitude decreases when the released vortices are convected away from the surface. This suggests the existence of a critical level of vorticity which must accumulate in order for separation to occur.

To demonstrate the growth of the shear layer perturbation in time and space in Fig. 4(a), the instantaneous shear layers from three consecutive snapshots are depicted in maroon, red, and pink, respectively. In this sequence, the local peaks and valleys in the shear layer are convected downstream with each consecutive time step.

To determine the instantaneous shear layer amplitudes in the velocity field snapshots, a datum reference for the shear layer is required. Due to the transient nature of the flow development during separation and reattachment, the datum reference varies in time. To determine the instantaneous reference for the shear layer, a sliding temporal average of the shear layer is taken. The average shear layer line was calculated based on 10 snapshots ( 5 before and 5 after) corresponding to a time window of $10 \mathrm{~ms}$. This time window was selected to preserve the transient nature of the flow development while

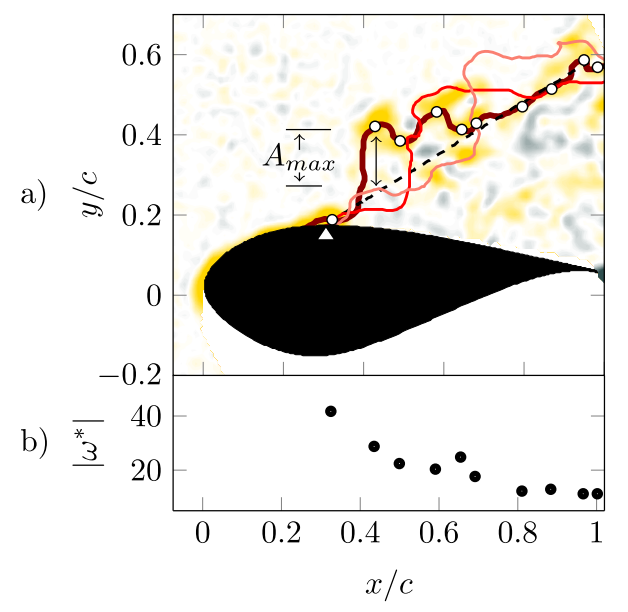

FIG. 4. (a) Example of the estimated shear layer line during reattachment. Three sequential shear layers are depicted for $\tau$ (dark red), $\tau_{1}$ (red), and $\tau_{2}$ (light red) along with the linear fit of the moving averaged shear layer (black dashed line). The shear layer amplitude is given by $A$. (b) Normalized vorticity maxima $\left|\omega^{*}\right|$ along the shear layer plotted as a function of the chordwise coordinate $(x / c)$. 
allowing sufficient convergence of the reference shear layer line. To further reduce the effect of outliers in the sliding average, the averaged shear layer lines were linearly fitted. The fits are calculated based on the 26 data points along the average shear layer line that are close to the airfoil surface. The 26 data points correspond to a spatial length of approximately $0.2 c$ extending away from the shear layer origin. The linear fits of the sliding averaged shear layer lines were then used as the datum reference to calculate the shear layer amplitudes in the individual snapshots. It is exemplarily depicted by the black dashed line in Fig. 4.

The shear layer amplitude $A(x / c)$ is the vertical distance between the estimated shear layer line and linear fit of the moving averaged shear layer and is exemplarily presented in Fig. 5 for the vorticity snapshot of Fig. 4. The peak in the amplitude closest to the separation point is denoted by $A_{1}$ and the maximum amplitude by $A_{\max }$.

Using the techniques from the work of Melius et al. ${ }^{16}$ the flow development stages within the stall cycle are identified. The separation point $f$, which is determined by locating the first occurrence of a negative streamwise velocity near the airfoil surface, is plotted in green as a function of time in Fig. 6. The intervals that capture stall development and reattachment are highlighted in gray.

To evaluate the trend of the vorticity accumulation at the point of separation, the first vorticity peak along the shear layer is extracted from all snapshots. In Fig. 6, the first four statistical moments of the first vorticity peak downstream of the separation point are presented. The top plot includes the separation point (green), the separation point vorticity, the angle of attack (gray) for reference, and the mean vorticity at the point of separation (black) as a function of convective time $\tau=t U_{\infty} / c$. Mean values are calculated for each of the identified stall stages (delineated by vertical dashed lines). The time intervals describe the duration of each stage of the stall cycle. ${ }^{16}$ The first time scale is the reaction time, $t_{r}$. This time interval begins with the initial motion of the blade, and the end is marked by a dramatic change in behavior of the separation point. The second time interval is termed the stall development time scale and is represented by $t_{s d}$. The beginning of this stage is marked by the emergence of stall development and a change in the separation point and ends when the primary stall vortex is shed, corresponding to a second dramatic

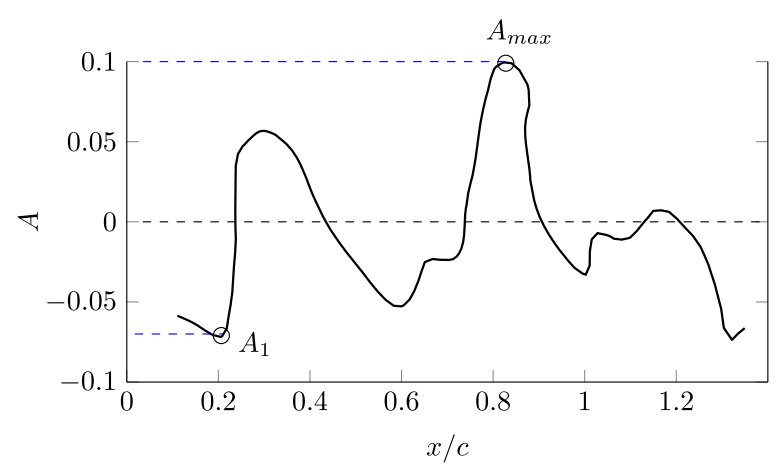

FIG. 5. Example of the shear layer amplitude $A(x / c)$ as a function of the chordwise position for the vorticity snapshot presented in Fig. 4. The first local maximum $A_{1}$ and the global maximum amplitude $A_{\max }$ are highlighted.

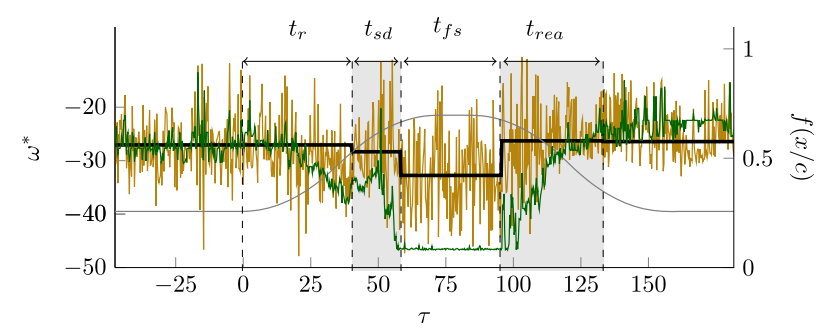

FIG. 6. Temporal evolution of the peak vorticity at the point of separation (gold), the separation point (green), and the motion profile (gray).

change in the separation point. The change marks the beginning of the next interval, identified as the period of time that the blade is experiencing full stall, $t_{f s}$. The phase ends when the angle of attack is reduced and the flow begins to reattach. During this process, the separation point moves downstream away from the leading edge. The fourth and final time interval is the time required for the flow to reattach to the surface and is termed the reattachment time scale $t_{\text {rea }}$. The mean value during attached flow, prior to separation, is $\left|\bar{\omega}^{*}\right|=25.9$. The mean increases by $8 \%$ to $\left|\bar{\omega}^{*}\right|=28.0$ during stall development. Once the flow enters full stall, the vorticity peak increases to $\left|\bar{\omega}^{*}\right|=32.6$, a $25 \%$ increase from the attached flow mean. The peak vorticity drops to $\left|\bar{\omega}^{*}\right|=25.5$ during flow reattachment. After the stall cycle is complete, the attached flow vorticity is the lowest of the calculated means at $\left|\bar{\omega}^{*}\right|=25.1$. As the angle of attack increases, the vorticity accumulates within a smaller region relative to the accumulated vorticity region at lower angles.

Alternatively, the circulation of the small-scale vortex close to the point of separation is analyzed. The circulation is calculated by the integral of the vorticity within a $\lambda_{2}=0$ contour which indicates the location and size of a vortical region. The separation point circulation refers to the circulation associated with the vortex nearest to the separation point. In Fig. 7, the non-dimensionalized separation point circulation $\left[\Gamma^{*}=\Gamma /\left(U_{\infty} c\right)\right]$, which refers to the circulation associated with the vortical structure nearest to the separation point, is presented in blue. This figure also includes the separation point (green) and the mean stage-wise separation point circulation (black) as a function of time to provide reference.

Prior to the blade motion, circulation at the point of separation is shown to fluctuate around $\left|\Gamma^{*}\right|=2.3$. Once the blade starts to pitch up and the separation point moves toward the leading edge, the stage-wise average value slightly increases to $\left|\bar{\Gamma}^{*}\right|=2.7$. The circulation during the pitching up motion

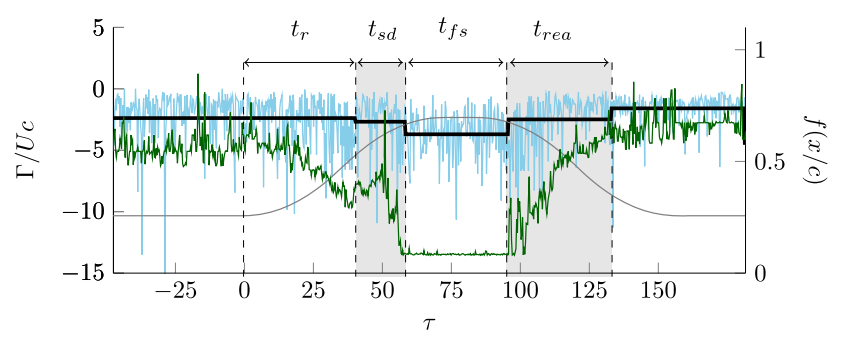

FIG. 7. Temporal evolution of the circulation at the point of separation (blue), the separation point (green), with the mean values of each stage (black), and the motion profile (gray). 
increases due to the change in the chordwise pressure gradient, which causes fluid near the surface to slow down. The influence of the pressure gradient is identified by a general increase in circulation at higher angles of attack. The largest values of circulation at the separation point are found during the full stall stage where $\left|\bar{\Gamma}^{*}\right|=3.7$. After the blade starts to pitch down, the mean circulation at the separation point drops again to $\left|\bar{\Gamma}^{*}\right|=2.5$ and even falls below its pre-stall values to $\left|\bar{\Gamma}^{*}\right|=1.6$ after flow reattachment.

The behavior of the vorticity and circulation at the separation point follow the same trend. During the attached flow, their absolute values are relatively low. When transition to the separated flow starts, they both increase to adjust for the changing surface pressure gradient, and at the point of full stall, a maximum is reached.

To highlight the perturbation behavior during unsteady separation, the tortuosity of the instantaneous shear layer is presented in Fig. 8. Tortuosity is defined as the ratio of the length of the shear layer relative to the distance it covers. In the case of a straight line, the tortuosity would be $T=L / D=1$. It follows that a value of $\mathrm{T}$ greater than 1 represents a perturbed shear layer. The tortuosity of each shear layer from the stall cycle is presented in Fig. 8 (green). In the pre-stall attached flow, there is a clear periodic peak in $T$ as it varies between a minimum of $T \approx 1$ and peaks of $T=1.6-1.75$. The time between successive peaks is approximately $\tau=10$. Considering shear layer perturbations as indications of vortex shedding, the time scale between peaks of $T$ is converted to the shear layer Strouhal number, $S t^{*}=c /\left(\Delta \tau U_{\infty}\right)=0.12$, consistent with results found in Ref. 19.

It is shown that the growth of shear layer tortuosity leads to the advancement of the separation point. During the attached flow stage, increased tortuosity corresponds to the movement of the separation point toward the leading edge, with peaks of tortuosity occurring during separation point advancement and troughs occurring when the separation point is retreating. The trend continues even when there is no periodic behavior any more. Once the pitching motion starts, the tortuosity increases in volatility and the periodic pattern observed previously is no longer present. The largest average values of $T$ are found in the stall development stage as the flow passes through the most unstable state transitioning to full stall. The shear layer is particularity tortuous during the moments just before full stall is reached. During full stall, the average tortuosity increases, which corresponds to the increase in relative size of the separation region. It is also noted that $T$ is relatively constant

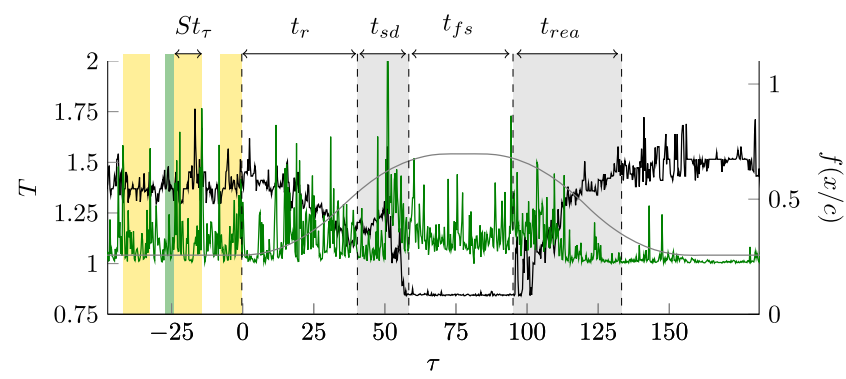

FIG. 8. Time history of the shear layer tortuosity (green), separation point (black), and pitching motion (gray). throughout this stage. As the reattachment stage begins, there is a spike in the tortuosity and the variability of $T$ increases. There is a significant drop off in $T$ near $\tau=120$ at which point the tortuosity of the shear layer approaches unity and rarely departs for the remaining duration of the time series. It appears that the shear layer directly after reattachment is more stable than that during the equilibrium state at the start of the pitching motion. The downstream convection of the excess wake fluid that occurs during reattachment thus seems to stabilize the reattached flow. ${ }^{20,21}$

Figure 9 provides a deeper look into the peak amplitude of the shear layer perturbation as a function of chordwise position. The shear layer amplitude $A(x / c)$ for individual snapshots is calculated by the vertical distance between the shear layer and the linear fit of the moving averaged shear layer. The maximum amplitude $A_{\max }$ is presented in Fig. 9 by the color of the marker while the position of the markers indicated the chordwise location at which the maximum amplitude is observed. The values of $A_{\max }$ are normalized by the chord. Dark green represents large amplitudes, and yellow represents small amplitudes. The peak amplitudes are plotted together with the angle of attack, $\alpha(\tau)$, in gray and the separation point, $f(\tau)$, in black. The figure shows the relative stability of the shear layer. When the angle of attack is held constant, the amplitudes of the shear layer perturbations grow with the downstream distance, following the general principles of the Kelvin-Helmholtz instability. For example, during the period of time prior to the beginning of the pitching motion, the amplitudes nearest to the point of separation are consistently small, $A_{\max } \approx 0.01$, while the perturbations near the trailing edge have grown to $A_{\max } \approx 0.1$ in some cases. As the angle of attack increases and stall development begins, the occurrence of a large $\Delta f$ is accompanied by a large perturbation amplitude that occurs close to the point of separation. This phenomenon is shown between $50 \leq \tau \leq 60$ during the largest movements of the separation point. Once the separation process has reached full stall, the maximum amplitudes primarily reside near the trailing edge. As the angle of attack decreases, the amplitude of the shear layer perturbation grows to values exceeding $A_{\text {max }}=0.15$ and begins to move toward the leading edge. As the flow reattaches, the values of the shear layer peak amplitude drop to values of less than $A_{\max }=0.05$. During this stage, the shear layer roll-up and shedding of the surface vorticity lead to large perturbations at the point of separation. When the vorticity on the surface is shed, the resulting shear layer perturbation is large at the point of separation. It is

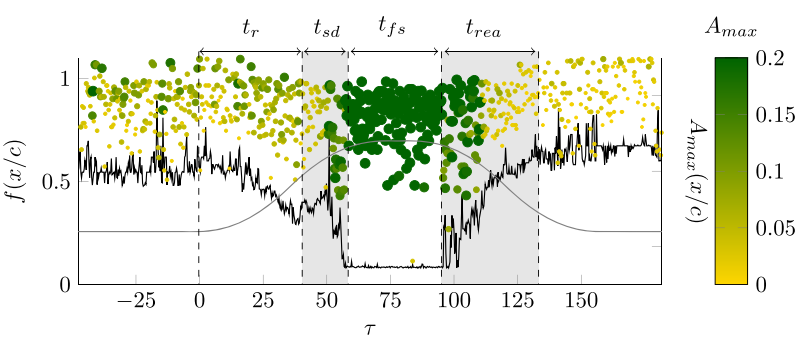

FIG. 9. Time history of the location of the maximum perturbation together with the separation point and pitching motion. The maximum perturbation amplitude is color-coded. 
worth noting that the peak amplitudes of the reattached flow are smaller than what is seen in the attached flow prior to separation.

Figure 9 demonstrates that large shear layer perturbations are present near the separation point during stall development and reattachment. To further explore this, the shear layer perturbation peak closest to the point of separation, $A_{1}$, is analyzed in Fig. 10. It is hypothesized that this particular perturbation will directly influence the movement of the separation point, according to Ref. 14. The separation point movement increases when perturbations at the separation point are larger than $A_{1} \approx 0.05$. This is most apparent during the initial attached flow, stall development, and initial portion of reattachment, $95 \leq \tau \leq 115$. During full stall and the final attached phase, the perturbation amplitudes are considerably smaller, indicating the relative stability of the shear layer during those periods. The combination of these results demonstrates that the formation and shedding of a consistent quantity of vorticity at the point of separation has direct influence on the shear layer behavior and perhaps most importantly on the movement of the separation point location.

The relationship between the change in the separation point and the strength of vorticity at the surface is tested for the Granger causality. ${ }^{22}$ Granger causality test results produce F-statistics that imply physical causality. Owing to the specific limitations of a Granger test to stationary signals and that the results are limited to only two of the possible dependent variables in the system, the Granger test does not necessarily imply direct physical causation. However, with a positive test result, it is expected that predictive models for the dependent variable benefit from information included in the independent variable.

Two series of Granger tests are conducted to reinforce the conclusions discussed in Sec. II. First, a Granger test is preformed to determine if the separation point location results in a change of the peak vorticity. When testing the null hypothesis that the location point does not Granger cause the peak vorticity amplitude, a p-value of $10^{-15}$ is the result. The confidence interval being quite small, the null hypothesis is rejected and it can be said that information about the separation point location will improve predictions of the peak surface vorticity at the point of separation. Next, the null hypothesis that the vorticity value nearest to the separation point does not Granger cause movement in the separation point is tested. The resulting $\mathrm{p}$-value for this test is $10^{-5}$; thus, the null hypothesis is rejected

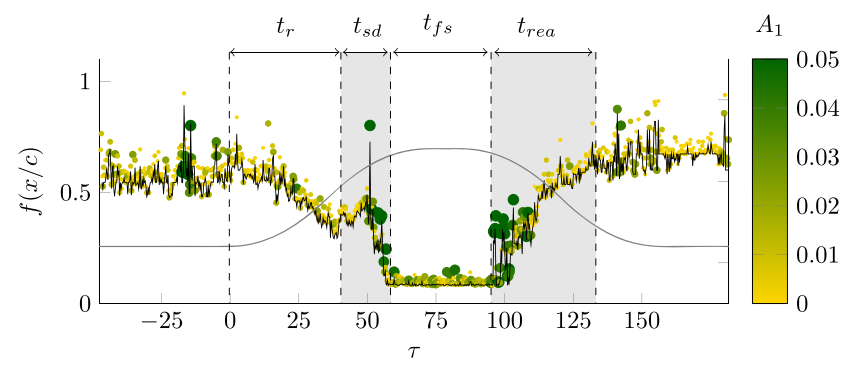

FIG. 10. Time history of the separation point perturbation location, the separation point, and the pitching motion. The separation point perturbation amplitude is color-coded. and it can be said that the vorticity value at the surface will help to predict the movement of the separation point.

\section{CONCLUSIONS}

Using time-resolved PIV, the role of surface vorticity and its accumulation on the unsteady movement of the separation point during flow separation is examined. The spatiotemporal evolution of the shear layer is characterized by the perturbation amplitude and local maxima of the vorticity along the estimated shear layer. The detection of the shear layer is based on a contour of the velocity at $0.48 U_{\infty}$. By evaluating the magnitude of vorticity accumulation at the separation point, there is a critical threshold value of vorticity that is consistently identified at the point of separation. This value is repeatedly found to be $\left|\omega^{*}\right| \approx 20$ for the presented test conditions. Above this value, the accumulated vorticity entrains enough fluid near the surface to create flow reversal and move away from the surface. The presented observation of a critical threshold of surface vorticity prior to vortex shedding will hopefully encourage follow-up investigations focusing on an extension of the parameter range, the universality of this observation, and the variation of the exact value of the vorticity threshold as a function of airfoil geometry and flow conditions.

This result also opens interesting prospects and opportunities to control the extent of separation by influencing the vorticity accumulation over an airfoil surface. It is also observed that immediately following its departure from the surface, the vortex begins to lose strength following an exponential decay. The changes in local vorticity peak values are correlated with the change in angle of attack. As the angle of attack is increased, the mean peak vorticity increases. Thereafter, it remains large during full stall and reduces in magnitude as the angle of attack decreases and flow begins to reattach. This trend is directly related to the angle dependent change in the chordwise pressure gradient, which at higher angles of attack limits the area where the accumulation occurs, leading to a higher circulation.

The shear layer development during unsteady separation is characterized by the perturbation amplitude and tortuosity. Tortuosity provides a global perspective on the contortion of the shear layer, which is subjected to the Kelvin-Helmholtz instability during the stall cycle. It is shown that the growth of shear layer tortuosity leads to the advancement of the separation point. During the attached flow, increased tortuosity is shown to correspond to the movement of the separation point toward the leading edge, with peaks of tortuosity occurring during separation point advancement and troughs occurring when the separation point is retreating. The periodic nature of this interaction is linked to the vortex shedding frequency, quantified by a Strouhal number of $S t^{*}=0.12$. The values of $T$ are consistently larger during stall development, full stall, and reattachment, owing to the unstable nature of the transitions and convective instability of the shear layer during these stall stages. Looking deeper into the unsteady shear layer behavior, two amplitudes are quantified during the stall cycle. The perturbation maximum location is captured revealing that perturbation amplitudes grow as they are convected downstream 


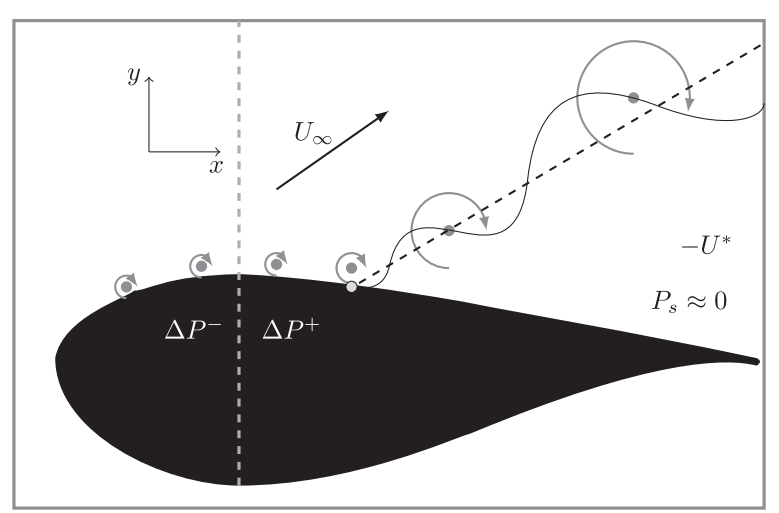

FIG. 11. Sketch of a shear layer extending away from a surface. The perturbation amplitude is influenced by the presence of vortices which have shed and are being convected away.

according to the Kelvin-Helmholtz instability theory. A fundamental description of this behavior is provided in Fig. 11. A shear layer (solid black line) extending away from a surface is influenced by the presence of vortices. As they are convected downstream, the perturbation amplitudes and vortex diameters grow as the vorticity strength diffuses.

The spatial occurrence of the maximum amplitudes during unsteady separation and reattachment is shown to approach the separation point. The results show that during the stages of stall development and reattachment the shear layer amplitudes are considerably larger than those during the more stable full stall and attached flow states.

The combination of these results demonstrates that the formation and shedding of a consistent quantified amount of vorticity at the point of separation directly influences the shear layer behavior and the movement of the separation point location. The movement of the separation point is recognized as a discrete process which is influenced by the dynamic interaction of a changing pressure gradient and the time dependent accumulation and shedding of surface vorticity.

By influencing the formation and shedding of the peak vorticity through surface based vorticity control mechanisms, one could limit the negative impact of the shear layer instability on the movement of the separation point. Such vorticity control would reduce the unsteady loading caused by unsteady separation. These results serve to reinforce the theory of unsteady separation by providing a quantification of the interaction between the accumulation of surface vorticity and the role of the shear layer during unsteady separation.

\section{ACKNOWLEDGMENTS}

This work was supported by the National Science Foundation, Grant No. NSF-CBET-1034581.

${ }^{1}$ D. F. Young and F. Y. Tsai, "Flow characteristics in models of arterial stenoses-II. Unsteady flow," J. Biomech. 6, 547-559 (1973).

${ }^{2}$ J. Song, H. Luo, and T. L. Hedrick, "Three-dimensional flow and lift characteristics of a hovering ruby-throated hummingbird," J. R. Soc., Interface 11, 20140541 (2014).

${ }^{3} \mathrm{~K}$. Cassel and A. Conlisk, "Unsteady separation in vortex-induced boundary layers," Philos. Trans. R. Soc., A 372, 20130348 (2014).

${ }^{4}$ L. Prandtl, "Über flüssigkeitsbewegung bei sehr kleiner reibung," in Internationalen Mathematiker-Kongresses, Heidelberg, Leipzig, 1904.

${ }^{5} \mathrm{~S}$. Goldstein, "On laminar boundary-layer flow near a position of separation,” Q. J. Mech. Appl. Math. 1, 43-69 (1948).

${ }^{6} \mathrm{~F}$. K. Moore, "On the separation of the unsteady laminar boundary layer," in Grenzschichtforschung/Boundary Layer Research (Springer, 1958), pp. 296-311.

${ }^{7}$ N. Rott, "Unsteady viscous flow in the vicinity of a stagnation point," Q. Appl. Math. 13, 444-451 (1956).

${ }^{8}$ W. R. Sears, "Some recent developments in airfoil theory," J. Aeronaut. Sci. 23, 490-499 (1956).

${ }^{9}$ L. L. van Dommelen and S.-F. Shen, "The spontaneous generation of the singularity in a separating laminar boundary layer," J. Comput. Phys. 38, 125-140 (1980).

${ }^{10}$ A. Surana, G. B. Jacobs, O. Grunberg, and G. Haller, "An exact theory of three-dimensional fixed separation in unsteady flows," Phys. Fluids 20, 107101 (2008).

${ }^{11}$ M. Farazmand and G. Haller, "Computing Lagrangian coherent structures from their variational theory," Chaos 22, 013128 (2012).

${ }^{12}$ A. Prasad and C. H. K. Williamson, "The instability of the shear layer separating from a bluff body," J. Fluid Mech. 333, 375-402 (1997).

${ }^{13}$ D. Tritton, Physical Fluid Dynamics (Oxford Science Publication, Clarendon Press, 1988).

${ }^{14}$ S. K. Roberts and M. I. Yaras, "Large-eddy simulation of transition in a separation bubble," J. Fluids Eng. 128, 232-238 (2006).

${ }^{15} \mathrm{~J}$. Wu, R. Tramel, F. Zhu, and X. Yin, "A vorticity dynamics theory of three-dimensional flow separation," Phys. Fluids 12, 1932-1954 (2000).

${ }^{16}$ M. Melius, R. B. Cal, and K. Mulleners, "Dynamic stall of an experimental wind turbine blade," Phys. Fluids 28, 034103 (2016).

${ }^{17}$ L. Graftieaux, M. Michard, and N. Grosjean, "Combining PIV, POD and vortex identification algorithms for the study of unsteady turbulent swirling flows,” Meas. Sci. Technol. 12, 1422-1429 (2001).

${ }^{18} \mathrm{P}$. Huerre and P. A. Monkewitz, "Absolute and convective instabilities in free shear layers,” J. Fluid Mech. 159, 151-168 (1985).

${ }^{19}$ S. Yarusevych, P. E. Sullivan, and J. G. Kawall, "On vortex shedding from an airfoil in low-Reynolds-number flows," J. Fluid Mech. 632, 245-271 (2009).

${ }^{20}$ R. A. M. Galbraith, M. W. Gracey, and E. Leitch, "Summary of pressure data for thirteen airfoils on the University of Glasgow's aerofoil database," technical report, University of Glasgow, 1992.

${ }^{21}$ W. Sheng, R. A. M. Galbraith, and F. N. Coton, "Return from aerofoil stall during ramp-down pitching motions," J. Aircraft 44, 1856 (2007).

${ }^{22} \mathrm{H}$. Y. Toda and T. Yamamoto, "Statistical inference in vector autoregressions with possibly integrated processes," J. Econometrics 66, 225-250 (1995). 